\title{
Review of AdS/CFT Integrability, Chapter I.2: The spectrum from perturbative gauge theory
}

\author{
C. SIEG
}

Institut für Mathematik und Institut für Physik, Humboldt-Universität zu Berlin Johann von Neumann Haus, Rudower Chaussee 25, 12489 Berlin, Germany

Niels Bohr International Academy, Niels Bohr Institute

Blegdamsvej 17, 2100 Copenhagen, Denmark

csieg@math.hu-berlin.de

\begin{abstract}
We review the constructions and tests of the dilatation operator and of the spectrum of composite operators in the flavour $S U(2)$ subsector of $\mathcal{N}=4 \mathrm{SYM}$ in the planar limit by explicit Feynman graph calculations with emphasis on analyses beyond one loop. From four loops on, the dilatation operator determines the spectrum only in the asymptotic regime, i.e. to a loop order which is strictly smaller than the number of elementary fields of the composite operators. We review also the calculations which take a first step beyond this limitation by including the leading wrapping corrections.
\end{abstract}




\section{Introduction}

In the context of the AdS/CFT correspondence [1], the discovery of integrability is a key ingredient towards finding the exact spectrum of strings in $\mathrm{AdS}_{5} \times \mathrm{S}^{5}$ and of composite operators in $\mathcal{N}=4 \mathrm{SYM}$ theory with gauge group $S U(N)$ in the planar limit, i.e. for $N \rightarrow \infty$. As reviewed in chapters [II.1] and [II.2], on the string side of the duality the spectrum is accessible order by order as a strong coupling expansion in terms of the 't Hooft coupling by a (semi)classical analysis of string states with large quantized charges. It is also described in terms of respective string Bethe ansätze which are reviewed in chapter [III.1].

In the $\mathcal{N}=4 \mathrm{SYM}$ theory, the weak coupling expansion of the planar spectrum, i.e. the conformal dimensions of composite operators, can be obtained by direct perturbative calculations of various correlation functions. The appearance of UV divergences requires renormalization, which then leads to a mixing among operators with the same bare conformal dimension. The eigenvalues of the new eigenstates under conformal rescalings are given as the sum of the bare scaling dimension and an individual anomalous dimension. The operator mixing can be extracted, e.g. from the correlation functions involving two composite operators. Alternatively, one can directly calculate the diagrams which contribute to the renormalization of these operators. This directly allows one to obtain an expression for the dilatation operator, whose eigenvalues are the anomalous dimensions.

Perturbative calculations become very cumbersome at high loop orders and can be avoided, if the observed integrability at one loop, which is reviewed in chapter [I.1], also persists to higher loop orders. The dilatation operator can then be determined, using some very general structural information from the underlying Feynman graphs only and some data from the gauge Bethe ansätze. The details of this approach are reviewed in chapter [I.3]. Direct Feynman graph calculations of the dilatation operator in the flavour $S U(2)$ subsector to three loops and of some of its eigenvalues and of parts of the Bethe ansätze also to higher loops provide important checks for the assumed integrability.

Even if integrability holds to all loop orders, the respective Bethe ansätze and planar dilatation operator allow us to compute the anomalous dimensions only in the asymptotic regime. In this regime, the loop order of the result is constrained to be strictly smaller than the length (the number of elementary fields) of the shortest composite operator involved. At loop orders which are equal to or exceed this number, the so-called wrapping interactions [2,3] have to be considered. They are corrections due to the finite size of the composite operators and have their origin in the neglected higher genus contributions to the dilatation operator [4]. In the dual string theory the counterparts of the wrapping interactions are corrections due to the finite circumference of the closed string worldsheet cylinder [5]. Their analyses are reviewed in chapters [III.5] and [III.6].

In this chapter we review the explicit Feynman graph calculations in $\mathcal{N}=4 \mathrm{SYM}$ theory in the planar limit beyond one loop. It is organized as follows:

In Section 2 we give a short summary of how composite operators are renormalized, and how the dilatation operator is defined in terms of the renormalization constants.

In Section 3 we then review the explicit calculations and tests of the dilatation oper- 
ator with particular focus on calculations beyond the first order in perturbation theory. ${ }^{1}$ Only the flavour $S U(2)$ subsector will be considered, since most higher loop calculations are performed within this subsector. As examples we recalculate in detail the respective one- and two-loop dilatation operator in $\mathcal{N}=1$ superfield formalism. This approach is much more efficient than the originally used formalism without manifest supersymmetries, and it yields more direct relations between the dilatation operator and the underlying Feynman graphs. We then display the result of a three-loop calculatoin and also summarize the existing checks of the magnon dispersion relation, of the structure of the dilatation operator and of some of its eigenvalues in the asymptotic regime at three and higher loops.

In Section 4, we review the perturbative calculations which consider the first wrapping corrections and hence yield results beyond the asymptotic regime. The general strategy of these calculations will be explained. In this way, the four-loop anomalous dimension for the length four Konishi descendant in the flavour $S U(2)$ subsector could be determined. Further results for different operators and for the terms of highest transcendentality are then summarized briefly.

In Section 5 we give a concluding summary, and in two appendices we present the explicit D-algebra manipulations for the one- and two-loop calculation and the expressions for the relevant integrals.

\section{Renormalization of composite operators}

The dilatation operator and anomalous dimensions can be obtained from a perturbative calculation of the correlation functions which involve the composite operators $\mathcal{O}_{a}$, where $a$ labels the different operators. The encountered UV divergences require a renormalization of the composite operators as

$$
\mathcal{O}_{a, \text { ren }}\left(\phi_{i, \text { ren }}\right)=\mathcal{Z}_{a}{ }^{b}(\lambda, \varepsilon) \mathcal{O}_{b, \text { bare }}\left(\phi_{i, \text { bare }}\right), \quad \phi_{i, \text { ren }}=\mathcal{Z}_{i}^{1 / 2} \phi_{i, \text { bare }},
$$

where in an appropriate basis $\mathcal{Z}=\mathbb{1}+\delta \mathcal{Z}$, and the matrix $\delta \mathcal{Z}$ is of order $\mathcal{O}(\lambda)$ in the renormalized coupling constant $\lambda$. It also depends on the regulator $\varepsilon$ and is in general non-diagonal and thus leads to mixing between the different composite operators. The matrix element $\delta \mathcal{Z}_{a}{ }^{b}$ is given by the negative of the sum of the overall UV divergences of the Feynman diagrams in which the vertices of the theory lead to interactions between the elementary fields of operator $\mathcal{O}_{b}$, such that the resulting external field flavour and ordering coincide with the ones of the operator $\mathcal{O}_{a}$. One also has to consider contributions from wave function renormalization of the elementary fields $\phi_{i}$ the operators are composed of. Respective factors $\mathcal{Z}_{i}^{1 / 2}$ are included within $\mathcal{Z}$.

$\mathcal{N}=4 \mathrm{SYM}$ theory can be regularized by supersymmetric dimensional reduction 6 in $D=4-2 \varepsilon$ dimensions. The coupling constant $g_{\mathrm{YM}}$ is then accompanied by the 't Hooft mass $\mu$ in the combination $g_{\mathrm{YM}} \mu^{\varepsilon}$ to restore the mass dimension of the loop integrals. Thereby, $g_{\mathrm{YM}}$ is not renormalized and hence itself does not depend on $\mu$, such that superconformal invariance is preserved. This was explicitly found to three loops by

\footnotetext{
${ }^{1}$ The one-loop results are reviewed in chapter $[.1$.
} 
computing the vanishing of the $\beta$-function in an $\mathcal{N}=1$ superfield formulation $[7]$. The finiteness of $\mathcal{N}=4 \mathrm{SYM}$ theory was then later shown to all orders [8]. A first argument was given in $[9]$. In particular, the self-energy of the superfields is finite, i.e. $\mathcal{Z}_{i}^{1 / 2}$ is trivial. ${ }^{2}$ In the planar limit, where the coupling constant is $\lambda=g_{\mathrm{YM}}^{2} N$, the dilatation operator is then extracted from the renormalization constant of the composite operators in $(2.1)$ as

$$
\mathcal{D}=\mu \frac{\mathrm{d}}{\mathrm{d} \mu} \ln \mathcal{Z}\left(\lambda \mu^{2 \varepsilon}, \varepsilon\right)=\lim _{\varepsilon \rightarrow 0}\left[2 \varepsilon \lambda \frac{\mathrm{d}}{\mathrm{d} \lambda} \ln \mathcal{Z}(\lambda, \varepsilon)\right] .
$$

The logarithm of $\mathcal{Z}=\mathbb{1}+\delta \mathcal{Z}$ has to be understood as a formal series in powers of $\delta \mathcal{Z}$. All poles of higher order in $\varepsilon$ must cancel in $\ln \mathcal{Z}$, such that it only contains simple $\frac{1}{\varepsilon}$ poles. In effect, the above description extracts the coefficient of the $\frac{1}{\varepsilon}$ pole of $\mathcal{Z}$, and at a given loop order $K$ multiplies it by a factor $2 K$. This then yields the dilatation operator as a power series

$$
\mathcal{D}=\sum_{k \geq 1} g^{2 k} \mathcal{D}_{k}, \quad g=\frac{\sqrt{\lambda}}{4 \pi},
$$

where for later convenience we have absorbed powers of $4 \pi$ into the definition of a new coupling constant $g$.

\section{Dilatation operator in the $S U(2)$ subsector}

$\mathcal{N}=4$ SYM theory contains six real scalar fields, four complex Weyl fermions and a gauge field that all transform in the adjoint representation of the gauge group $S U(N)$. In the following we denote these fields as components fields, since in a superspace formalism they appear as components of superfields. In order to build the $\mathcal{N}=1$ superfields, the real scalar component fields are complexified and combined together each with one fermion or with its complex conjugate into three chiral superfields $\phi_{i}, i=1,2,3$ or respectively anti-chiral ones $\bar{\phi}_{i}$. The three field flavours are transformed into each other by an $S U(3)$ subgroup of the $S U(4)$ R-symmetry group. The remaining gauge field and fermions are combined together into an $\mathcal{N}=1$ vector superfield $V$. An explicit expression of the $\mathcal{N}=4 \mathrm{SYM}$ action in terms of $\mathcal{N}=1$ superfields and the respective Feynman rules in which the Wick rotation is included can be found, e.g. in [10]. The superspace conventions are as in [11], where also an introduction to the D-algebra is given. The latter is required to reduce the supergraphs, i.e. the Feynman diagrams in superspace, to ordinary spacetime objects that are located at a single point in the fermionic coordinates of superspace.

\subsection{Operator mixing in the $S U(2)$ subsector}

In the following, we denote the three chiral field flavours of $\mathcal{N}=4$ SYM theory by $\phi_{i}=(\phi, \psi, Z)$. The flavour $S U(2)$ subsector contains operators which are composed of only two different types of these fields, e.g. $\phi$ and $Z$. Their color indices are all contracted

\footnotetext{
${ }^{2}$ This holds apart from gauge artefacts that are not relevant here.
} 
with each other to yield a gauge invariant object. In general, the gauge contractions form several cycles, and one obtains a multi-trace operator. Such an operator is a normalordered product of single-trace operators, i.e. of operators each of which only contains a single cycle of gauge contractions.

Mixing only occurs between those operators that have the same numbers of both types of fields $\phi$ and $Z$. Then, it suffices to consider operators which contain a number of fields $\phi$ that does not exceed the number of fields $Z$, since the results for the remaining operators follow immediately by an exchange of the role of the two fields. Usually, the fields $\phi$ are denoted as impurities which appear between fields of type $Z$ within the traces over the gauge group. Furthermore, in the planar limit that we exclusively consider from now on $]^{3}$ the Feynman diagrams that alter the gauge trace structure of the composite operators are suppressed. The renormalization of multi-trace operators then follows immediately from the one of their single-trace constituents. We can therefore restrict the analysis to single-trace operators. In this case, the planar Feynman diagrams can only affect the ordering of the two different types of fields inside the single trace, but they cannot alter their multiplicities and in particular the length $L$ of the composite operators that is defined as the total number of constituent fields. Flavour contractions cannot appear, since the composite operators of the $S U(2)$ subsector do not contain the complex conjugate fields $(\bar{\phi}, \bar{\psi}, \bar{Z})$. The $S U(2)$ subsector is closed under renormalization, at least perturbatively $[12]$. The operators

$$
\operatorname{tr}\left(Z^{L}\right), \quad \operatorname{tr}\left(\phi Z^{L-1}\right)
$$

which are the ground state and a state with a single impurity are protected and do not acquire anomalous dimensions. Operators which contain more than a single impurity $\phi$ undergo non-trivial mixing.

Since the aforementioned operator mixing only occurs within subsets of single-trace operators that only differ by permutations of their field content, the renormalization constant $\mathcal{Z}$ and hence also the dilatation operator $\mathcal{D}$ can be expressed in terms of flavour permutations that act on the constituent fields of these composite operators. The flavour permutations themselves can be written as products of permutations acting on nearest neighbour sites. For composite operators of fixed length $L$ they are given by $[13$

$$
\left\{a_{1}, \ldots, a_{n}\right\}=\sum_{r=0}^{L-1} \mathrm{P}_{a_{1}+r a_{1}+r+1} \cdots \mathrm{P}_{a_{n}+r a_{n}+r+1}
$$

and by the identity \{\} in flavour space that measures the length $L$ of the composite operator it is applied to. The structures consider the insertion of the Feynman subdiagrams in which elementary fields interact at all possible positions within the single trace of the composite operator by the summation. Periodicity with period $L$ is thereby understood. No other insertions have to be considered here, since in the planar limit the interactions have to occur between adjacent fields.

The permutation structures (3.2) admit a definition of the range of the interaction in flavour space obtained from their lists of arguments as

$$
\kappa=\max _{a_{1}, \ldots, a_{n}}-\min _{a_{1}, \ldots, a_{n}}+2 .
$$

\footnotetext{
${ }^{3}$ See chapter IV.1] for a review concerning effects of non-planarity.
} 
The range $\kappa$ and hence also the possible arguments $a_{1}, \ldots, a_{n}$ of the permutation structures are subject to constraints from the underlying Feynman diagrams. In order to find the restrictions for those structures that can appear in the expression of the dilatation operator, we focus on Feynman diagrams in which the elementary interactions occur in a single region that is simply connected also when the composite operator is removed from the diagram. These diagrams may have overall UV divergences that contribute with simple $\frac{1}{\varepsilon}$ poles to the renormalization constant $\mathcal{Z}$ and hence according to (2.2) also to the dilatation operator. The remaining diagrams, in which the elementary interactions occur in several non simply-connected regions after the removal of the composite operator, cannot contribute with simple $\frac{1}{\varepsilon}$ poles. Their calculation is only required if one wants to determine $\mathcal{Z}$ itself completely, for example in order to check explicitly that in $\ln \mathcal{Z}$ all higher order poles in $\varepsilon$ cancel. Here, we will not consider them further and only focus on the diagrams that can contribute to the dilatation operator. The interaction range $R$ of a diagram of the latter type is defined as the number of adjacent elementary fields of the composite operator that enter the single simply connected interaction region. It can only yield contributions with permutation structures (3.2) that obey the following conditions:

$$
n \leq K, \quad \kappa \leq R, \quad R \leq K+1,
$$

where $K$ denotes the number of loops inside the diagram. The first inequality considers that each nearest-neighbour permutation is associated with at least one loop. The second condition ensures that the range of the interaction in flavour space does not exceed the interaction range $R$ of the Feynman diagram. In a third inequality $R$ itself is bounded from above by the loop order, since each interaction between nearest neighbour fields of the composite operator generates at least one loop. We denote the diagrams that saturate this bound, i.e. the ones with interaction range $R=K+1$ as maximum range diagrams. Since the summation in (3.2) runs over all insertion points with periodicity $L$, the smallest integer entry can always be fixed, e.g. to 1 by shifting all $a_{i}$ by a common integer. According to (3.4) the biggest integer can then be at most $K$. Further relations between the structures (3.2) can be found in [14]. The independent permutation structures which obey (3.4) then form a basis in which the $K$-loop dilatation operator can be written down.

The basis with elements (3.2) is not the best choice in order to express the result of an explicit Feynman diagram calculation, since the different flavour arrangements within a single Feynman diagram generate linear combinations of several permutation structures (3.2) with fixed relative coefficients. If, instead, the generated combinations themselves are used as basis elements, each Feynman diagram is associated with only one of them $[15,16]$. The basis elements obtained from supergraphs are called chiral functions and are defined as

$$
\chi\left(a_{1}, \ldots, a_{n}\right)=\left.\left\{a_{1}, \ldots, a_{n}\right\}\right|_{\mathrm{P} \rightarrow \mathrm{P}-\mathbb{1}},
$$

where $\mathrm{P} \rightarrow \mathrm{P}-\mathbb{1}$ denotes a replacement of all permutations in $(3.2)$ by the fixed combination of permutation and identity. The expansion of the resulting products yields $\chi$ in terms of linear combinations of permutation structures. For each $\chi$ we define the range of the interaction in flavour space by applying the definition (3.3) to its list of 
arguments. The chiral functions capture the structure of the chiral and anti-chiral superfield lines of the underlying supergraphs. Hence, all supergraphs which only differ by the arrangement of the flavour-neutral vector fields generate contributions with the same chiral function. In particular, at loop order $K$ the chiral functions $\chi\left(a_{1}, \ldots, a_{n}\right)$ with $n=K$ are associated each with a single Feynman graph since they do not contain any vector fields. We denote the respective graphs as chiral graphs.

Except of the identity $\chi()=\{\}$, all chiral functions (3.5) yield zero when they are applied to one of the protected states in (3.1). The expression of the dilatation operator in terms of chiral functions should hence not explicitly depend on $\chi()$. We will come back to this statement at the end of Section 3.5.2.

\subsection{One-loop dilatation operator}

The one-loop calculation in the $S U(2)$ subsector was addressed by Berenstein, Maldacena and Nastase in 17$]$. They used component fields to compute the term involving the permutation structure $\{1\}$, which permutes the flavour of two neighbouring fields. It is the maximum shuffling term at one loop, since it shifts the position of the impurity by the maximum number of one site at this loop order. Its generalization to higher loops will be discussed in Section 3.5.1. The remaining Feynman diagrams all contribute to the identity operation \{\} in flavour space and were not computed explicitly. Instead, their contribution was reconstructed from the fact that the eigenvalue for the ground state in (3.1) should be zero. Furthermore, the contributions in which two neighbouring impurities interact with each other were neglected.

Using $\mathcal{N}=1$ superfields instead of component fields for the one-loop calculation, only a single Feynman diagram contains a UV divergence and hence contributes to the renormalization constant in $(2.1)$. It is evaluated as

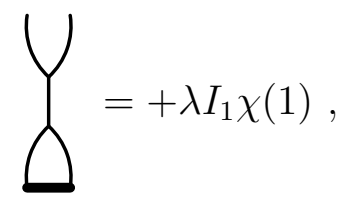

where the bold horizontal line represents the composite operator of arbitrary length $L \geq 2$, thereby omitting its $L-2$ elementary field lines that do not participate in the local interaction. The D-algebra manipulations are trivial in this case as explicitly displayed in Appendix A. The resulting loop integral is given in Appendix B. The further oneloop diagram of gluon exchange is finite, and the one-loop wave function renormalization vanishes. This is different from their behaviour in component formalism, where they have to be considered. According to the description (2.2), the one-loop dilatation operator follows from $(3.6)$ as

$$
\mathcal{D}_{1}=-2 \chi(1)
$$

Including also the contributions to the trace operator in flavour space, which extends the result to the flavour $S O(6)$ subsector $4^{4}$ the full one-loop calculation in component fields was performed in [18], and the result was recognized as the Hamiltonian of a respective integrable Heisenberg spin chain.

\footnotetext{
${ }^{4}$ The flavor $S O(6)$ subsector is only closed to one loop.
} 


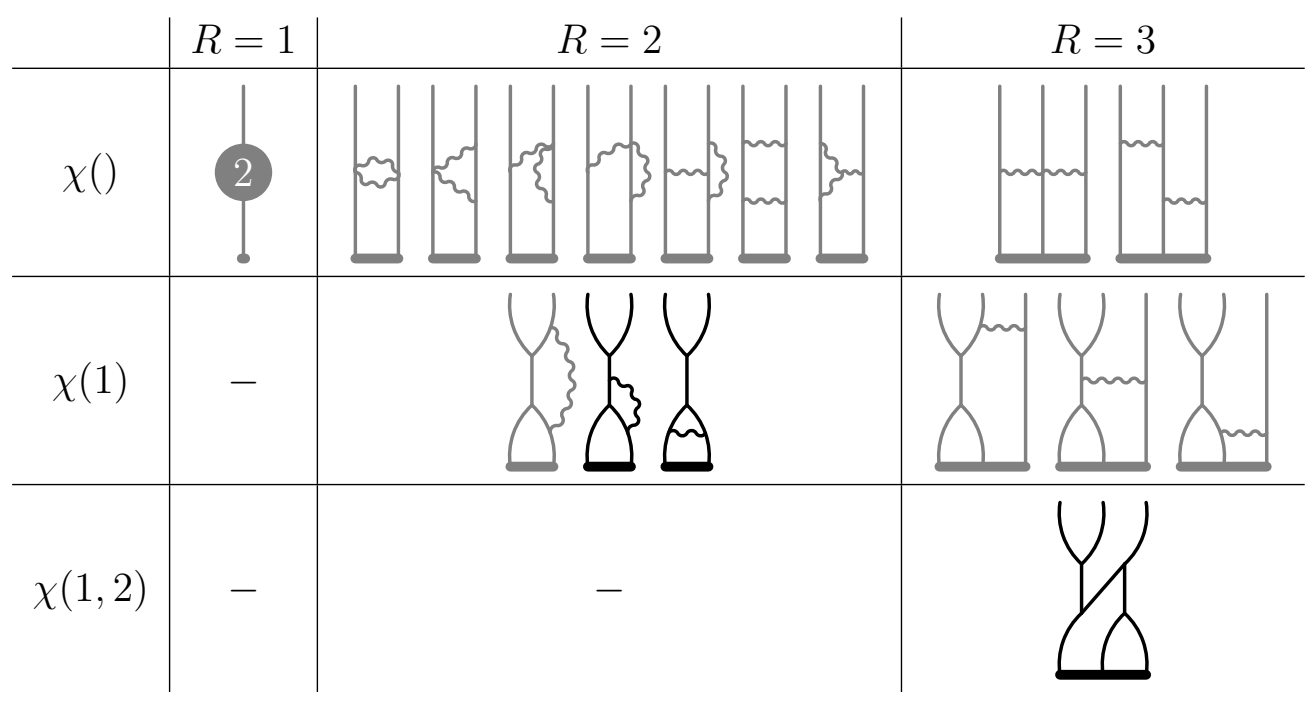

Table 1: Diagrams in $\mathcal{N}=1$ superfields (apart from eventual reflections) which can in principle contribute to the two-loop dilatation operator. Graphs which contain the vanishing one-loop self-energies are not drawn. It turns out that all diagrams depicted in gray are also irrelevant. The two-loop chiral self-energy is finite, and the remaining range $R \geq 2$ diagrams are irrelevant due to generalized finiteness conditions [10].

\subsection{Two-loop dilatation operator}

A two-loop renormalization of composite operators in the $S U(2)$ subsector was performed in [19] in component formalism. As in the one-loop case [17] only the diagrams which contribute to genuine flavour permutations were explicitly calculated, and the coefficient of the identity operation was determined by the condition of a vanishing eigenvalue of the ground state (3.1). Furthermore, the contributions in which impurities interact with each other were neglected.

The relevant diagrams for the complete two-loop calculation of the dilatation operator in terms of $\mathcal{N}=1$ superfields are given in Table 1. The chiral self-energy is identically zero at one loop and finite at higher loops. According to the generalized finiteness conditions derived in $[10]$, all range $R \geq 2$ diagrams, in which all vertices appear in loops are also finite. This concerns all remaining diagrams in the first line and in the second line the respective first diagram in the second and third columns. The pole parts of the last two diagrams in this line in the third column cancel against each other [15,16]. This cancellation is based on the fact that, in order to obtain contributions with overall UV divergences, a sufficient number of spinor derivatives $\mathrm{D}_{\alpha}$ and $\overline{\mathrm{D}}_{\dot{\alpha}}$ has to remain inside the loops in order to be transformed into spacetime derivatives. This yields constraints on the D-algebra manipulations that amount to the formulation of generalized finiteness conditions in [10]. All diagrams that are irrelevant due to these conditions are depicted in gray. We only have to compute the remaining diagrams and consider also their reflections where necessary. The substructures in the relevant range $R=2$ diagrams with chiral function $\chi(1)$ combine into the one-loop chiral vertex correction that is explicitly given 
in $(\mathrm{A} .2)$. We then find

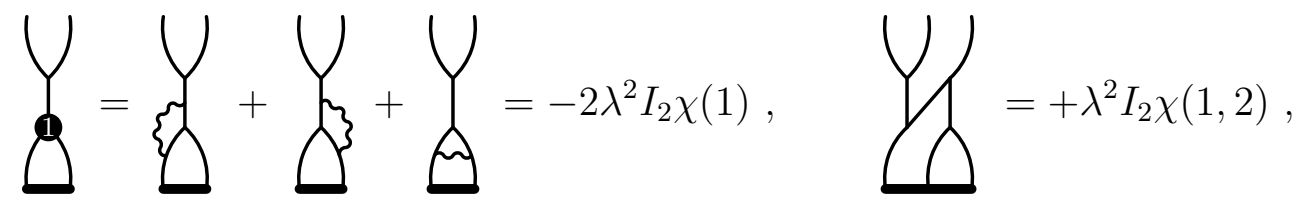

where we have to consider also the reflection of the last diagram which contributes with chiral function $\chi(2,1)$. According to the description $(2.2)$, the two-loop dilatation operator is then obtained by extracting the $\frac{1}{\varepsilon}$ pole of the sum of these diagrams and multiplying it by -4 . With the pole part of the respective integral $I_{2}$ given in (B.4) this then yields

$$
\mathcal{D}_{2}=4 \chi(1)-2[\chi(1,2)+\chi(2,1)] .
$$

An explicit demonstration of the cancellation of the double poles in $\ln \mathcal{Z}$ as mentioned after $(2.2)$ can be found in [10], where the one- and two-loop calculations were presented as a demonstration for the efficiency of the used approach.

\subsection{Three-loop dilatation operator}

At three-loop order a calculation of the dilatation operator directly from Feynman graphs of $\mathcal{N}=1$ superfields was recently performed in $[10]$. The result reads

$$
\begin{aligned}
\mathcal{D}_{3}= & -4(\chi(1,2,3)+\chi(3,2,1))+2(\chi(2,1,3)-\chi(1,3,2))-4 \chi(1,3) \\
& +16(\chi(1,2)+\chi(2,1))-16 \chi(1)-4(\chi(1,2,1)+\chi(2,1,2)) .
\end{aligned}
$$

It determines the planar spectrum in the $S U(2)$ subsector to three loops and hence goes beyond an earlier test of two eigenvalues [20], which employs Anselmi's trick [21] to reduce the calculation to two loops. The three-loop results confirm the prediction from integrability in [13]. Earlier checks of some of the three-loop eigenvalues are summarized in Section 3.5.3.

\subsection{Partial tests at higher loops}

To three-loop order and also beyond, certain parts of the respective Bethe ansatz and dilatation operator have been checked by direct Feynman diagram calculations. This concerns the so-called maximum shuffling terms, which contribute to the dispersion relation of the Bethe ansatz. Further terms in the higher loop expressions of the dilatation operator have also been tested explicitly.

\subsubsection{Tests of the magnon dispersion relation}

Even if with the assumed integrability the $S U(2)$ dilatation operator itself has been determined only to the first few loop orders (see chapter [I.3] for a review), the magnon dispersion relation of the Bethe ansatz is an all-order expression and directly related to certain Feynman diagrams. For a single magnon of momentum $p$ it is given by 3$]$

$$
E(p)=\sqrt{1+16 g^{2} \sin ^{2} \frac{p}{2}}-1
$$


and it is fixed by the underlying symmetry algebra up to an unknown function of the coupling constant $[22]$, which in the $\mathcal{N}=4$ SYM case essentially appears to be given by $g^{2}$ itself and has already been substituted accordingly. ${ }^{5}$

At a fixed loop order $K$ in the expansion of the above relation, the momentum dependence can be expressed as linear combination of the elements $\cos (k-1) p \sin ^{2} \frac{p}{2}$ with $1 \leq k \leq K$. In particular, the term with $k=K$ is generated by the so-called maximum shuffling diagrams, which include shifts of the position of a single impurity (which is a magnon in the spin chain notation) by the maximum number of $K$ neighbouring sites. The relevant diagrams are given by

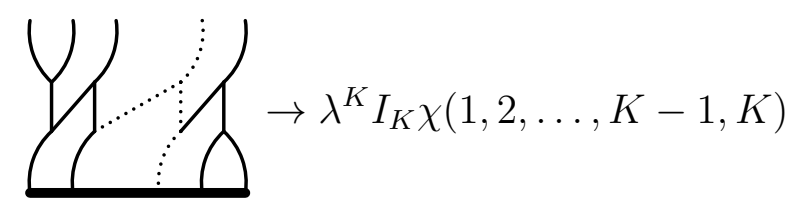

and by its reflection. When the sum of these two diagrams is applied to the eigenstate of a single magnon with momentum $p$, it yields the eigenvalue

$$
\lambda^{K} I_{K}[\chi(1,2, \ldots, K)+\chi(K, \ldots, 2,1)] \rightarrow-8 \lambda^{K} I_{K} \cos (K-1) p \sin ^{2} \frac{p}{2} .
$$

According to the description (2.2), the $\frac{1}{\varepsilon}$ pole of this expression has to be multiplied by $-2 K$ to obtain its contribution to the magnon dispersion relation. A comparison with the respective term in the expansion of $(3.11)$, thereby taking into account the relation (2.3) between the couplings, then makes a prediction for the $\frac{1}{\varepsilon}$ pole of the integral $I_{K}$ as

$$
\operatorname{Res}_{0}\left(\mathrm{KR}\left(I_{K}\right)\right)=\frac{1}{(4 \pi)^{2 K}} \frac{(2 K-2) !}{(K-1) ! K !} \frac{1}{K} .
$$

The explicit expressions for the poles of $I_{K}$ for some $K$ are listed in (B.4). They are consistent with this result.

In [23] it was shown that at generic loop order the pole structure of the maximum shuffling diagrams in component fields is in accord with the BMN square root formula [17]. The latter was proposed as an all-order expression for the anomalous dimensions in the so-called BMN limit, where the length $L$ of the operators and the coupling $g$ become infinite $L, g \rightarrow \infty$, thereby keeping fixed the numbers of impurities inside the operators and also the effective coupling constant $g^{\prime}=\frac{g}{L}$. For magnon momenta $p_{j}=\frac{2 \pi n_{j}}{L} \ll 1$ the dispersion relation (3.11) yields the individual contributions of each magnon $j$ with mode number $n_{j}$ to the BMN square root formula. Since the scattering of magnons is neglected, their momenta $p_{j}$ assume a simple form and are solutions of the originally proposed Bethe equations [3] with a magnon S-matrix that becomes trivial in the BMN limit. However, these Bethe equations do not yield the anomalous dimensions of $\mathcal{N}=4 \mathrm{SYM}$ theory since the S-matrix is incomplete. One has to consider the so-called dressing phase [24] that first appeared at strong coupling $[25]$ but is important also at weak coupling $[24,26]$, where it alters the magnon momenta at order $\mathcal{O}\left(g^{6}\right) \cdot{ }^{6}$ Due to the dressing phase, the

\footnotetext{
${ }^{5}$ The explicit Feynman diagram calculation in 10 confirms that this is correct to three loops. It is non-trivial in the $\mathrm{AdS}_{4} / \mathrm{CFT}_{3}$ correspondence that is reviewed in chapter IV.3.

${ }^{6}$ The dressing phase is reviewed in chapter [III.3].
} 
S-matrix violates perturbative BMN scaling, i.e. its perturbative expansion diverges if after the replacement $g \rightarrow g^{\prime} L$ the limit $L \rightarrow \infty$ is taken, thereby keeping $g^{\prime}$ fixed and small. The Bethe equations involving this S-matrix then yield anomalous dimensions that violate perturbative BMN scaling from four loops on. However, the BMN square root formula obeys this scaling, and hence it cannot describe the anomalous dimensions of operators with two or more impurities beyond three loops. ${ }^{7}$ Since the dressing phase only affects the scattering of magnons, all tests and derivations of the BMN square root formula that rely on the calculation of phase shifts of a single magnon are insensitive to this failure and succeed. This concerns the previously mentioned all-order test of the maximum shuffling terms $[23$ and also an all order derivation employing the $\mathcal{N}=1$ superfield formalism [27|. It would be more appropriate to say that in these calculations the magnon dispersion relation in the BMN limit is obtained.

The magnon dispersion relation (3.11) describes the free propagation of one magnon. It it thus built up from all Feynman diagrams with chiral functions that do not yield a vanishing result when applied to the single magnon momentum eigenstate. The number of impurities of the composite operator sets an upper bound on the number of bubbles formed by two neighbouring lines of the composite operator inside the Feynman diagrams. Such a bubble appears for example in the lower right corner of the graph in $(3.12)$, and it vanishes unless the two involved field flavours are different. The diagrams contributing to the magnon dispersion relation hence must not contain more than one of these bubbles. This restricts their chiral functions to $\chi(1, \ldots, k)$ and $\chi(k, \ldots, 1)$ after the identities for the permutation structures $(3.2)$ found in [14] have been used to simplify the chiral functions, e.g. as $\chi(1,2,1)=\chi(2,1,2)=\chi(1)$ in the three loop result (3.10). Allorder expressions for the coefficients of these terms in the dilatation operator then follow directly from the magnon dispersion relation (3.11) and can be found in [10]. It should be stressed that the aforementioned contributions also yield non-vanishing results when additional magnons are present outside of the $k+1$ interacting legs. They therefore also contribute to the magnon S-matrix.

\subsubsection{Tests of magnon scattering}

The Feynman diagrams that vanish for a single magnon state, but are non-vanishing if two or more magnons are present within their respective interaction ranges, should exclusively be associated with the magnon S-matrix. Their contributions appear together with the ones of the aforementioned maximum and non-maximum shuffling terms in the dilatation operator. In the $S U(2)$ subsector they first show up at three-loops as the contribution with chiral function $\chi(1,3)$ in $(3.10) .8$ The further chiral functions $\chi(2,1,3), \chi(1,3,2)$ are also associated with magnon scattering, but they only appear in a combination that is associated with a similarity transformation, i.e. a change in the basis of operators $[13,14]$, that does not affect the eigenvalues.

As a more complicated example, we consider the four-loop dilatation operator. It

\footnotetext{
${ }^{7}$ This breakdown is independent of the general restriction of the Bethe ansatz to the asymptotic regime that requires a termination of the expansion at a loop order $K \leq L-1$ to avoid the wrapping corrections.

${ }^{8}$ A two-loop test of the S-matrix of the $S L(2)$ subsector can be found in 28 .
} 
can be determined from the underlying integrability as reviewed in chapter [I.3]. In the basis of the chiral functions $(3.5)$ it reads

$$
\begin{aligned}
\mathcal{D}_{4}= & +200 \chi(1)-150[\chi(1,2)+\chi(2,1)]+8\left(10+\epsilon_{3 a}\right) \chi(1,3)-4 \chi(1,4) \\
& +60[\chi(1,2,3)+\chi(3,2,1)] \\
& +\left(8+2 \beta+4 \epsilon_{3 a}-4 i \epsilon_{3 b}+2 i \epsilon_{3 c}-4 i \epsilon_{3 d}\right) \chi(1,3,2) \\
& +\left(8+2 \beta+4 \epsilon_{3 a}+4 i \epsilon_{3 b}-2 i \epsilon_{3 c}+4 i \epsilon_{3 d}\right) \chi(2,1,3) \\
& -\left(4+4 i \epsilon_{3 b}+2 i \epsilon_{3 c}\right)[\chi(1,2,4)+\chi(1,4,3)] \\
& -\left(4-4 i \epsilon_{3 b}-2 i \epsilon_{3 c}\right)[\chi(1,3,4)+\chi(2,1,4)] \\
& -\left(12+2 \beta+4 \epsilon_{3 a}\right) \chi(2,1,3,2) \\
& +\left(18+4 \epsilon_{3 a}\right)[\chi(1,3,2,4)+\chi(2,1,4,3)] \\
& -\left(8+2 \epsilon_{3 a}+2 i \epsilon_{3 b}\right)[\chi(1,2,4,3)+\chi(1,4,3,2)] \\
& -\left(8+2 \epsilon_{3 a}-2 i \epsilon_{3 b}\right)[\chi(2,1,3,4)+\chi(3,2,1,4)] \\
& -10[\chi(1,2,3,4)+\chi(4,3,2,1)]
\end{aligned}
$$

The coefficients $\epsilon_{i}, i=3 a, 3 b, 3 c, 3 d$ in the above result are not fixed by the construction and parameterize the previously mentioned similarity transformations. The coefficient $\beta$ is the leading term of the previously mentioned dressing phase. The magnon dispersion relation is encoded in the first two terms in the first line, the second line and the last line. The further contributions should be associated with magnon scattering. As the contributions from the maximum shuffling diagrams $(3.12)$ in the last line, also the other terms in the last four lines have chiral functions that saturate all the bounds in $(3.4)$. Hence, the underlying Feynman diagrams are chiral and of maximum range and their contributions can be calculated as easily as the one of the maximum shuffling terms (3.12).

The term in (3.15) with chiral function $\chi(2,1,3,2)$ only satisfies the first bound in (3.4), i.e. the underlying Feynman diagram is chiral but it is not of maximum range. It involves the leading coefficient $\beta$ of the dressing phase, which can be determined from an evaluation of the respective diagram

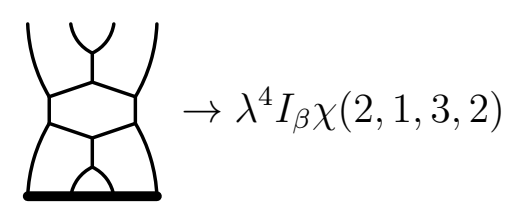

if the coefficient $\epsilon_{3 a}$ of the similarity transformations is known. One finds $\epsilon_{3 a}=-4$ for example by computing the diagram which generates $\chi(1,3,2,4)$ or $\chi(2,1,4,3)$. With the pole part of the integral $I_{\beta}$ given in $(\overline{B .5})$, the leading coefficient of the dressing phase is then determined as $\beta=4 \zeta(3)$. The result was obtained in [29], using component formalism. It agrees with one of the proposals in $[24]$ and with the result extracted from a four-loop calculation of a four-point amplitude in $[26]$.

It is also relatively easy to compute the terms with chiral functions which only saturate the second and third bound in (3.4), i.e. all terms in (3.15) with chiral functions that contain 1 and 4 in their lists of arguments and hence only stem from Feynman diagrams 
of maximum range $R=5$. This calculation was performed in $[15,16$ in $\mathcal{N}=1$ superfield formalism in the context of calculating the first wrapping correction to be discussed below. The results yield an overdetermined system of equations that uniquely fixes the coefficients $\epsilon_{i}$ and provides non-trivial checks of the remaining coefficients that are fixed by the underlying integrability. The analogous calculation of the $R=6$ diagrams at five loops can be found in [30].

The expressions $(\overline{3.7}),(\overline{3.9}),(\overline{3.10})$ and $(\overline{3.15})$ do not depend on the identity $\chi()$. This guarantees that the anomalous dimension of the BPS operators (3.1) are zero. The generalized finiteness conditions in $[10]$ predict this to all orders and relate it to the finiteness of the chiral self-energy, i.e. to the preservation of conformal invariance.

\subsubsection{Checks of eigenvalues}

To three loops the results $(3.7),(3.9)$ and $(3.10)$ for the dilatation operator have been obtained by direct Feynman diagram calculations. At higher loops, only the terms that saturate at least one of the bounds in (3.4) have been tested as described above. Further checks concern the eigenvalues of the dilatation operator for some composite operators. They should match with the anomalous dimensions obtained in direct Feynman diagram calculations.

Of particular interest is thereby the Konishi supermultiplet. As superconformal primary it contains the $\mathcal{N}=1$ Konishi operator [31] that has bare scaling dimension $\Delta_{0}=2$ and reads

$$
\mathcal{K}=\operatorname{tr}\left(\mathrm{e}^{-g_{\mathrm{YM}} V} \bar{\phi}_{i} \mathrm{e}^{g_{\mathrm{YM}} V} \phi^{i}\right) .
$$

This operator is not chiral, and hence all its superfield components lie beyond the $S U(2)$ subsector. However, the Konishi supermultiplet also contains an operator of this subsector. In order to find it, one has to select the level four descendant of bare dimension $\Delta_{0}=4$ that is chiral and pick out the relevant $S U(4)$ R-symmetry component given by

$$
\operatorname{tr}([\phi, Z][\phi, Z])
$$

It contains as lowest superfield component the respective operator built out of the two scalar fields of the flavour $S U(2)$ subsector.

All members of a superconformal multiplet acquire the same anomalous dimension. For the Konishi multiplet it is given to four loops in (4.1). The one- and two-loop contributions were obtained by explicit Feynman diagram calculations in [32] and [33], and then also by an OPE analysis in [34], see also [35]. These results are also found for a twist-two operator with conformal spin $S=2$ that appears within another level four descendant of the Konishi multiplet. It belongs to the closed $S L(2)$ subsector that contains certain operators with general twist and conformal spin $S$. For twist-two operators with generic $S$, the result to two loops has been obtained from Feynman diagrams in [36]. At three loops it could be extracted [37] as the terms with highest transcendentality, i.e. with highest degrees of the harmonic sums, from the NNLO QCD result for the non-singlet splitting functions of QCD [38]. The truncation of the QCD result is based on the observation [39] that due to special properties of the DGLAP and BFKL equations in $\mathcal{N}=4$ SYM theory a mixing between functions of different transcendentality degrees does not 
occur. Specializing to $S=2$, the extracted result agrees with the three-loop contribution in (4.1). When the dilatation operator given in (3.7), (3.9) and (3.10) is applied to the state (3.18), it also correctly yields the result in (4.1) $9^{9}$ In fact, the three-loop term was first predicted in [13], where the dilatation operator was constructed from integrability. Later, an explicit Feynman diagram calculation [20], which employs Anselmi's trick [21] to reduce the calculation to two loops, led to the same result. The calculation in 10 also confirms the result and furthermore fixes the planar three-loop spectrum of all composite single-trace operators of the flavour $S U(2)$ subsector from field theory by a direct Feynman diagram calculation of the dilatation operator.

The previously mentioned twist-two operators of the $S L(2)$ sector are very important for tests of the AdS/CFT correspondence and the underlying integrability. These tests are reviewed in chapter [III.4]. In particular, the results in the strict $S \rightarrow \infty$ limit are not modified by wrapping interactions. At finite $S$ such modifications occur. The simplest example is $S=2$, i.e. the operator which appears in the Konishi multiplet. Its anomalous dimension is affected by wrapping interactions at four loops and beyond.

\section{Wrapping interactions}

In the following we briefly summarize the calculations of the previously mentioned wrapping interactions. A more detailed review is given by [40].

The Bethe ansätze or the dilatation operator yield reliable results for the anomalous dimensions in the asymptotic limit only. The origin and precise form of this restriction can be understood by recalling the construction from Feynman diagrams. In Section 3 it was argued that at a given loop order $K$ the dilatation operator is determined from Feynman diagrams with range $R \leq K+1$, which lead to flavour permutations with range $\kappa \leq R$. For the construction of the diagrams, it is thereby implicitly assumed that the length $L$ of the involved composite operators is at least as big as the maximal interaction range $K+1$. Therefore, an application of the dilatation operator to composite operators of length $L$ can in general only yield the correct anomalous dimensions in the asymptotic limit, i.e. to a loop order $K \leq L-1$. At $K \geq L$ loops, the assumption of a sufficient length of the involved composite operators becomes invalid, and therefore contributions from diagrams with interaction range $R>L$ should be removed from the dilatation operator. Instead, there are contributions from new diagrams that are built with the operators of the respective lower length $L$. The new diagrams are called wrapping diagrams since, due to the insufficient length of the composite operators, the interactions wrap around them. Two examples of such diagrams are depicted in Figure 1. Beyond the asymptotic limit, the dilatation operator explicitly depends on the length $L$ of the composite operators it is applied to. More precisely, the coefficients of the chiral functions in the expression of the dilatation operator become functions of $L$ at loop orders $K \geq L$, while in the asymptotic limit they are constants, and the dilatation operator depends on the length only via the permutation structures $(3.2)$.

\footnotetext{
${ }^{9}$ At four and higher loops this is no longer the case since the wrapping interactions have to be considered. This will be discussed in Section 4 .
} 
The appearance of wrapping interactions is closely connected to the truncation of the genus $h$ expansion of the dilatation operator beyond the planar $h=0$ contribution [4]. If in a planar wrapping diagram the composite operator is replaced by a longer operator, the additional fields lines cannot leave the diagram without crossing any other lines, i.e. it becomes a diagram of genus $h=1$. The appearing wrapping diagrams hence come from certain genus $h=1$ contributions to the dilatation operator, which become planar when it is applied to a sufficiently short composite operator. Wrapping diagrams appear at all orders in the genus expansion of the dilatation operator. They are of genus $h+1$ in the asymptotic regime and encode the finite size effects at genus $h$. The planar wrapping diagrams are special since they can be projected out of all genus one contributions by introducing spectator fields [4]. While in general for higher genus diagrams the notion of the range of the interaction is not meaningful, it is still well defined for the subset of genus one diagrams when they become the planar wrapping diagrams. Integrability seems to persist, even if in general at higher genus its breakdown is expected [13].10

In order to obtain the anomalous dimensions beyond the asymptotic regime, one should not abandon the dilatation operator as obtained from the underlying integrability at loop orders $K \geq 4$ and compute all Feynman diagrams. Instead, the considerations at the beginning of this section imply that the dilatation operator is still useful, since it can be corrected for an application to composite operators of shorter length $L$. First, at each loop order $K$ all contributions from Feynman graphs of longer range $K+1 \geq R>L$ have to be removed. Then, contributions from the wrapping interactions have to be added.

This procedure is particularly powerful at the critical order $K=L$ where wrapping arises for the first time, since only relatively few Feynman diagrams of restricted topology have to be computed explicitly. Most diagrams are captured automatically by those terms in the dilatation operator that are not removed in the modification process. Also, the only contributions that one has to remove from the dilatation operator are the ones that come from Feynman diagrams with maximum range $R=K+1$. It is convenient to divide these diagrams according to their range of interaction in flavour space $\kappa$ into two classes. The first class contains diagrams with $\kappa=R=K+1$, i.e. according to the definition of $\kappa$ in (3.3) their range $R$ is encoded within the list of arguments of their chiral functions. The second class collects all the remaining diagrams with $\kappa<R=K+1$. Such Feynman diagrams contain a chiral structure with interaction range $\kappa$, and the remaining $R-\kappa$ neighbouring field lines are connected with it and with each other only by vector fields. Since the latter are flavour neutral, the range $R$ of these diagrams is not captured by the chiral functions. It was shown in $[16]$ in the $\mathcal{N}=1$ superfield formalism that the diagrams of the second class do not contribute to the dilatation operator: either they are finite or their overall UV divergences cancel against each other. This is also an implication of the generalized finiteness conditions derived in [10]. In Section 3.3 we have already used the results when we disregarded the two-loop diagrams with $R=3$ but $\kappa<3$ in the first two rows of the last column of Table 1. The diagrams of the first class that have $\kappa=R=K+1$ are the only maximum range diagrams that contribute with their overall UV divergences. These contributions can be easily identified and removed from the expression of the dilatation operator, since their chiral functions are of maximum

\footnotetext{
${ }^{10}$ In chapter IV.1 the analyses of higher genus contributions are reviewed.
} 
range. The subtraction procedure becomes almost trivial: one just has to remove all contributions with chiral functions that have 1 and $K$ within their list of arguments. This does not require the calculation of any Feynman diagrams. For example, in the four-loop expression (3.15) one removes the last contribution in the first line and the ones in the fifth, sixth and the last four lines. The eigenvalues of the subtracted dilatation operator are no longer independent of the scheme coefficients $\epsilon_{i}$, which have to be fixed by calculating at least some of the diagrams with range $R=K+1$. If one could compute the wrapping interactions that have to be added to the subtracted dilatation operator also as functions of $\epsilon_{i}$, the eigenvalues of the resulting operator should not depend on the $\epsilon_{i}$. However, the calculation of the wrapping interactions takes place in a scheme fixed by the use of $\mathcal{N}=1$ supergraphs, and therefore the $\epsilon_{i}$ in the subtracted dilatation operator have to assume the respective values. Finally, it is important to remark that the simplicity of the subtraction procedure is only guaranteed if chiral functions $(3.5)$ are used as basis elements. If, instead, the basis of permutation structures (3.2) is used, the subtraction of the contribution from a Feynman diagram with $R=K+1$ affects the coefficients of several permutation structures also with different flavour interaction ranges $\kappa \leq R$ in the dilatation operator ${ }^{11}$

The aforementioned method was first introduced and used in [15], with the details given in [16], in the case $K=L=4$, i.e. for the four-loop anomalous dimension of the Konishi operator. In $\mathcal{N}=4$ SYM theory it is the simplest case where wrapping arises. The calculation starts from the four-loop asymptotic dilatation operator (3.15) and modifies it for an application to the length four Konishi descendant of the flavour $S U(2)$ subsector $(3.18)$ in order to determine the correct eigenvalue [15, 16]. Including also the lower orders, the anomalous dimension of the Konishi operator to four-loops was then determined as

$$
\gamma=12 g^{2}-48 g^{4}+336 g^{6}+(-2496+576 \zeta(3)-1440 \zeta(5)) g^{8},
$$

where the full conformal dimension is obtained as $\Delta=\Delta_{0}+\gamma$ with the bare scaling dimension $\Delta_{0}$ as described in Section 3.5.3. The four-loop contribution has also been obtained from a generalized Lüscher formula [42]. This approach is reviewed in chapter [III.5]. Furthermore, it was later also found in a computer-based calculation in component formalism [43]. The matching of the Feynman diagram and Lüscher based calculations provides the first test of AdS/CFT and the underlying integrability beyond the asymptotic limit. It is also reproduced by the recently proposed $Y$-system [44], which is derived from the thermodynamic Bethe ansatz (TBA) [45] and is a candidate to capture the full planar spectrum of $\mathcal{N}=4$ SYM theory. The TBA and Y-system are reviewed, respectively, in chapters [III.6] and [III.7]. Earlier attempts to describe the wrapping effects in terms of integrable systems are included in chapter [I.3].

In $[46]$ the result $(4.1)$ which also holds for the earlier mentioned twist-two operator with conformal spin $S=2$ has been generalized to arbitrary $S$. When analytically continued to $S=-1$, it yields the correct pole structure as predicted from the BFKL equation.

\footnotetext{
${ }^{11}$ In the context of the BMN matrix model a subtraction attempt was made in 41 . It does not lead to the correct result, since the necessary modifications of the contributions with permutation structures of lower range and the addition of the wrapping diagrams was not performed.
} 
A result for the five-loop anomalous dimension of the Konishi operator has been obtained in impressive calculations on the basis of the generalized Lüscher formula [47] and the TBA [48]. Also this result has been generalized to arbitrary spin $S$, and it is in accord with the pole structure from the BFKL equation [49]. To obtain the five-loop result for the Konishi multiplet from a Feynman diagram calculation is very difficult, even with the universal cancellation mechanisms discovered in [10]. Instead, a five-loop result for the $L=5$ operator $\operatorname{tr}([\phi, Z][\phi, Z] Z)$ which is in the same supermultiplet as certain twist-three operators has been computed [30], and it agrees with the result from the generalized Lüscher formula $[50]$. The six-loop results for the twist-three operators with generic conformal spin $S$ has recently become available [51].

Beyond the asymptotic limit, the contributions of highest transcendentality, i.e. which contain the $\zeta$-function with biggest argument, are generated entirely by the wrapping interactions. In the four-loop result in (4.1) this is the term with $\zeta(5)$. Its generalization to twist-two operators with generic conformal spin $S$ has been obtained from a Feynman diagram calculation in component formalism in [52]. At generic loop and critical wrapping order $K=L$ the highest transcendentality degree of the wrapping diagrams is $2 K-3$ compared to $2 K-5$ of the dressing phase in the asymptotic Bethe ansatz. A clean setup that allows one to study the transcendentality structure without admixtures from the dressing phase is provided by single-impurity operators in the $\beta$-deformed $\mathcal{N}=4$ SYM theory. ${ }^{12}$ The leading wrapping corrections have been calculated up to 11 loops in [53] and were confirmed in [54]. A clear pattern emerges also for the terms of lower transcendentality. The diagrams in Figure 1 are responsible for the highest transcen-
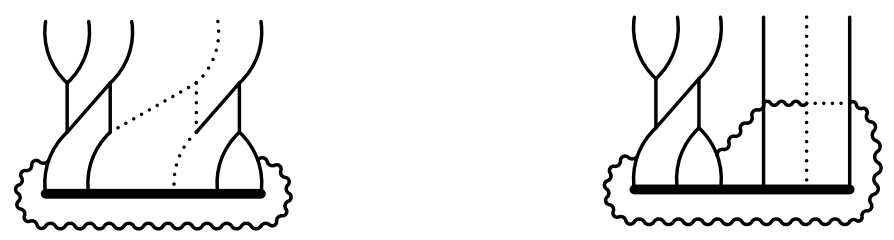

Figure 1: Wrapping diagrams that generate contributions of highest transcendentality at leading wrapping order.

dentality contribution involving $\zeta(2 K-3)$. The respective term can be traced back to a component $\frac{1}{2} P_{K}$ in the decomposition of the integrals, where $P_{K}$ is the $K$-loop cake integral given in (B.6).

\section{Conclusions}

We have reviewed the explicit Feynman diagram calculations which at small 't Hooft coupling determine the planar spectrum of composite operators in the flavour $S U(2)$ subsector of $\mathcal{N}=4 \mathrm{SYM}$ theory and test the underlying integrability. We have presented the calculations up to two loops in detail and summarized the calculations and partial checks at higher loops. The use of $\mathcal{N}=1$ superspace techniques and of chiral functions as operators in flavour space allowed us to directly interpret the Feynman diagrams in terms

\footnotetext{
${ }^{12}$ Among other deformations the $\beta$-deformation is reviewed in chapter IV.2].
} 
of the dispersion relation and the scattering matrix that appear in the integrability-based Bethe ansatz.

Then, we reviewed how anomalous dimensions beyond the asymptotic limit can be obtained by computing the leading wrapping corrections and which properties and interpretation these interactions have. The existing tests in these setups have been summarized.

\section{Acknowledgements}

I am very grateful to Francesco Fiamberti and Alberto Santambrogio for reading parts of the manuscript. I also want to thank Francesco Fiamberti, Matias Leoni, Andrea Mauri, Joseph Minahan, Alberto Santambrogio, Olof Ohlsson Sax, Gabriele TartaglinoMazzucchelli, Alessandro Torielli and Daniela Zanon for very pleasant collaborations on some of the papers reviewed here and in other chapters of this review.

\section{A D-algebra}

The propagators and vertices of superfields depend not only on the bosonic, but also on the fermionic coordinates $\theta^{\alpha}, \bar{\theta}^{\dot{\alpha}}$, of superspace and carry covariant spinor derivatives $\mathrm{D}_{\alpha}, \overline{\mathrm{D}}_{\dot{\alpha}}$. By the D-algebra manipulation which consists of transfers, partial integrations and the use of (anti)-commutation relations for products of these spinor derivatives, the underlying expression is transformed into the final result that is localized at a single point in the coordinates $\theta^{\alpha}, \bar{\theta}^{\dot{\alpha}}$. We refer the reader to $[11$ for an introduction to the $\mathcal{N}=1$ superfield formalism in the adopted conventions and to [10] for an explicit presentation of the relevant Feynman rules. Here, we only recall that two $\mathrm{D}_{\alpha}$ and two $\overline{\mathrm{D}}_{\dot{\alpha}}$ have to remain in each loop in order to obtain a non-vanishing result. The loop is then localized in the fermionic coordinates. We indicate this by filling it grey. Also, we recall two simple relations, $\mathrm{D}^{2} \overline{\mathrm{D}}^{2} \mathrm{D}^{2}=\square \mathrm{D}^{2}$ and $\overline{\mathrm{D}}^{2} \mathrm{D}^{2} \overline{\mathrm{D}}^{2}=\square \overline{\mathrm{D}}^{2}$, which transform spinor derivatives into spacetime derivatives $\square=\partial^{\mu} \partial_{\mu}$.

The one-loop diagram (3.6) requires no D-algebra manipulations, and one directly obtains

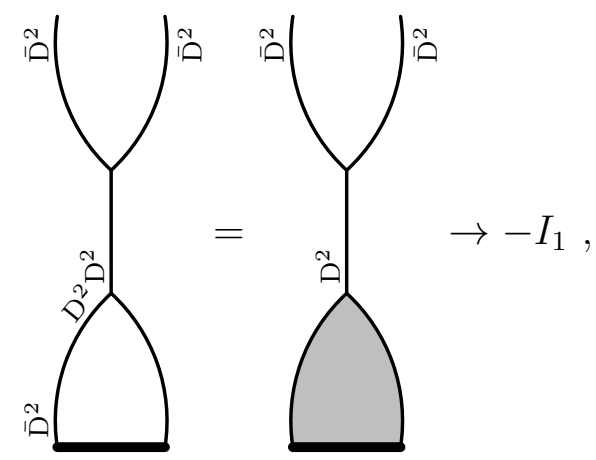

where the loop integral $I_{1}$, given in $(\overline{\mathrm{B} .2})$ for $K=1$, is the one extracted from the greyscaled region. Its UV pole is listed in (B.4). There appears an additional factor -1 in front of $I_{1}$ : we have to transform the full fermionic measure in the algebraic expression of 
the diagram into the chiral measure of the term that adds the chiral composite operator with a chiral source to the action. This means, we replace $\mathrm{d}^{4} \theta \rightarrow \mathrm{d}^{2} \overline{\mathrm{D}}^{2}$ and combine the extra derivatives $\bar{D}^{2}$ with the remaining $\mathrm{D}^{2}$ in the above diagram to $\square$, such that the propagator that connects the chiral and anti-chiral cubic vertex is cancelled, thereby yielding the factor -1 . In the result we have not considered any other non-trivial prefactors of the propagators and vertices. They are contained within the color- and flavour factors (chiral functions) of the complete result given in (3.6).

The one-loop correction to the chiral vertex that enters (3.8) is easily evaluated

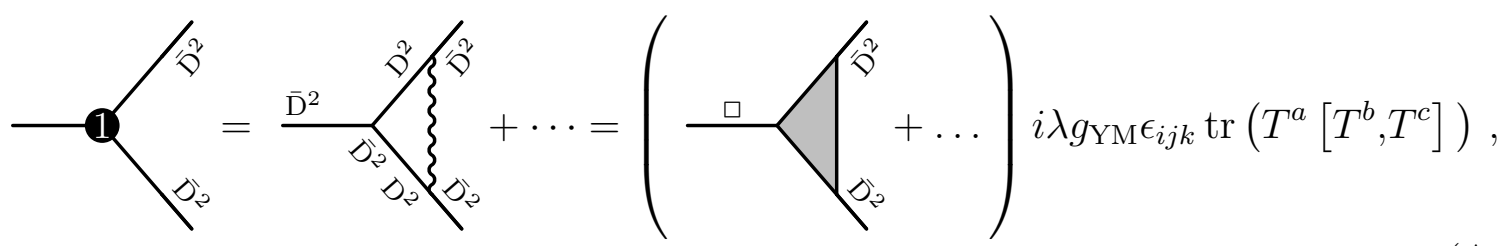

where the ellipsis denote the remaining two diagrams obtained by cyclic permutations of the external legs, and we have included the color and flavour factors. Also in this case, the $\square$ is produced after reducing the full fermionic measure to the chiral measure as mentioned above. When $\square$ cancels the propagator a factor -1 is produced.

The D-algebra manipulations for the diagrams (3.8) contributing to the two-loop dilatation operator are

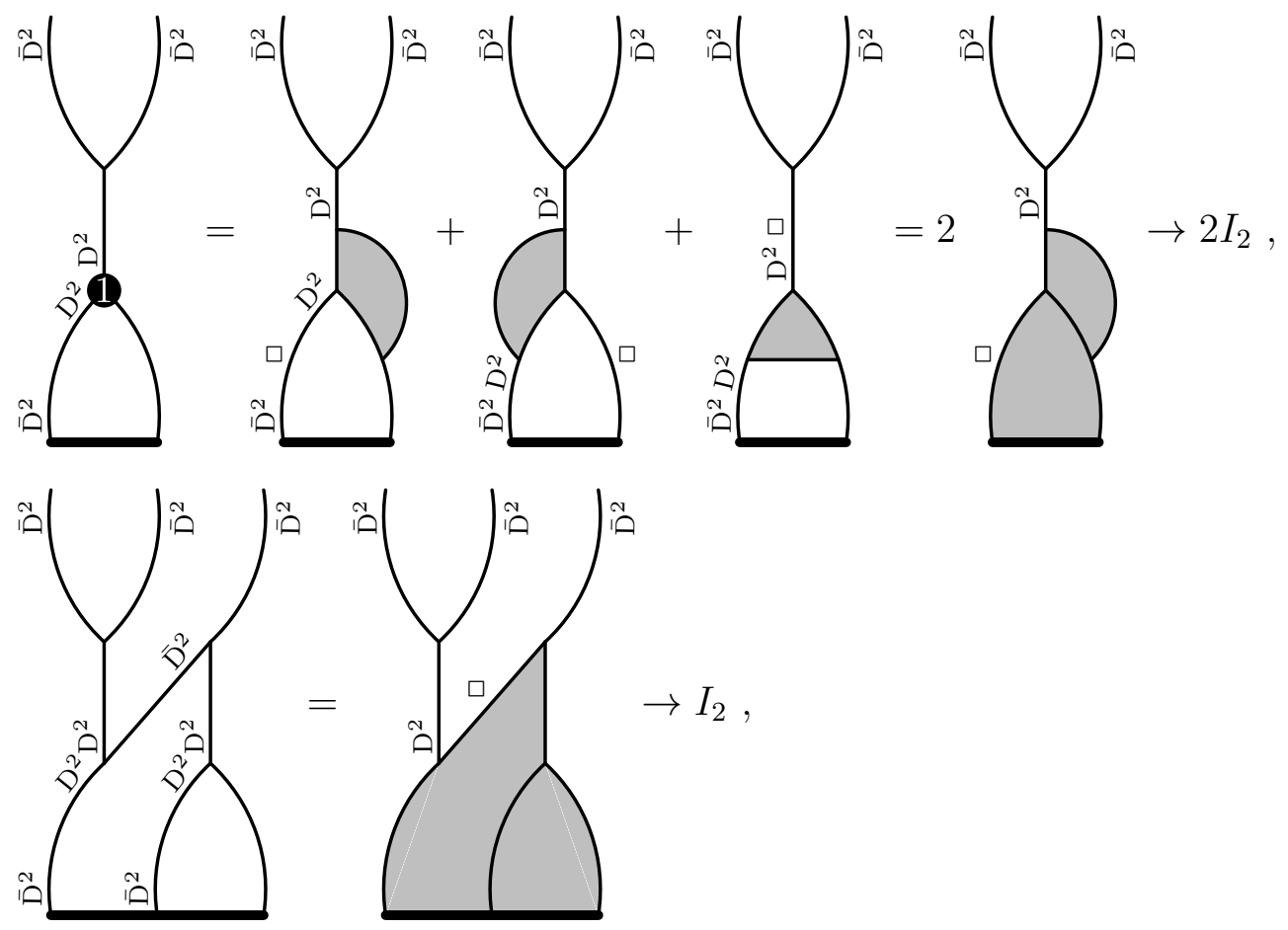

where equalities hold up to disregarded finite contributions, and the final expressions in terms of the integral $I_{2}$ consider the aforementioned factor -1 . 


\section{B Integrals}

Using the scalar $G$-function defined as

$$
G(\alpha, \beta)=\frac{\Gamma\left(\frac{D}{2}-\alpha\right) \Gamma\left(\frac{D}{2}-\beta\right) \Gamma\left(\alpha+\beta-\frac{D}{2}\right)}{(4 \pi)^{\frac{D}{2}} \Gamma(\alpha) \Gamma(\beta) \Gamma(D-\alpha-\beta)},
$$

in $D$-dimensional Euclidean space, the following integrals can be found exactly to all loop orders

$$
I_{K}=\underbrace{2}_{\vdots_{K-1}^{\prime} i_{i}^{\prime}}=\prod_{k=0}^{K-1} G\left(1-\left(\frac{D}{2}-2\right) k, 1\right) .
$$

They are logarithmically divergent in $D=4-2 \varepsilon$ dimensions, and their overall UV divergence is obtained with the operations $K$ to extract the pole part and $R$ to subtract subdivergences as

$$
\mathrm{KR}\left(I_{K}\right)=\mathrm{K}\left(I_{K}-\sum_{k=1}^{K-1} \mathrm{KR}\left(I_{k}\right) I_{K-k}\right) .
$$

To the first few loop orders, one finds

$$
\begin{aligned}
& \mathrm{KR}\left(I_{1}\right)=\frac{1}{(4 \pi)^{2}} \frac{1}{\varepsilon}, \\
& \mathrm{KR}\left(I_{2}\right)=\frac{1}{(4 \pi)^{4}}\left(-\frac{1}{2 \varepsilon^{2}}+\frac{1}{2 \varepsilon}\right) \\
& \mathrm{KR}\left(I_{3}\right)=\frac{1}{(4 \pi)^{6}}\left(\frac{1}{6 \varepsilon^{3}}-\frac{1}{2 \varepsilon^{2}}+\frac{2}{3 \varepsilon}\right) \\
& \mathrm{KR}\left(I_{4}\right)=\frac{1}{(4 \pi)^{8}}\left(-\frac{1}{24 \varepsilon^{4}}+\frac{1}{4 \varepsilon^{3}}-\frac{19}{24 \varepsilon^{2}}+\frac{5}{4 \varepsilon}\right), \\
& \mathrm{KR}\left(I_{5}\right)=\frac{1}{(4 \pi)^{10}}\left(\frac{1}{120 \varepsilon^{5}}-\frac{1}{12 \varepsilon^{4}}+\frac{11}{24 \varepsilon^{3}}-\frac{19}{12 \varepsilon^{2}}+\frac{14}{5 \varepsilon}\right), \\
& \mathrm{KR}\left(I_{6}\right)=\frac{1}{(4 \pi)^{12}}\left(-\frac{1}{720 \varepsilon^{6}}+\frac{1}{48 \varepsilon^{5}}-\frac{25}{144 \varepsilon^{4}}+\frac{47}{48 \varepsilon^{3}}-\frac{1313}{360 \varepsilon^{2}}+\frac{7}{\varepsilon}\right) .
\end{aligned}
$$

The pole parts of the integrals that appear in the calculations of the four-loop dressing phase or of the wrapping interactions at critical wrapping order can very efficiently be computed by using a modified and extended version of the Gegenbauer polynomial $x$ space technique $[55,16]$. The integral of the simplest contribution that allows us to determine the leading four-loop coefficient of the dressing phase reads

$$
I_{\beta}=\circlearrowleft, \quad K R\left(I_{\beta}\right)=\frac{1}{(4 \pi)^{8}}\left(-\frac{1}{12 \varepsilon^{4}}+\frac{1}{3 \varepsilon^{3}}-\frac{5}{12 \varepsilon^{2}}-\frac{1}{\varepsilon}\left(\frac{1}{2}-\zeta(3)\right)\right)
$$

The terms of highest transcendentality from wrapping corrections at critical order are determined by the cake integral. This integral is logarithmically divergent for $K \geq 3$ 
loops and reads

$$
P_{K}=K \overbrace{K-1}^{\prime}>_{4}^{3}, \quad \mathrm{~K}\left(P_{K}\right)=\frac{1}{(4 \pi)^{2 K}} \frac{1}{\varepsilon} \frac{2}{K}\left(\begin{array}{c}
2 K-3 \\
K-1
\end{array}\right) \zeta(2 K-3),
$$

where the pole part has been obtained in [56] at generic loop order.

\section{References}

[I.1] J. Minahan, "Review of AdS/CFT Integrability, Chapter I.1: Spin Chains in $\mathcal{N}=4$ $S Y M "$, arxiv:1012.3983.

[I.3] A. Rej, "Review of AdS/CFT Integrability, Chapter I.3: Long-range spin chains", arxiv:1012.3985.

[II.1] A. Tseytlin, "Review of AdS/CFT Integrability, Chapter II.1: Classical AdS $S_{5} \times S^{5}$ string solutions", arxiv:1012.3986.

[II.2] T. McLoughlin, "Review of AdS/CFT Integrability, Chapter II.2: Quantum Strings in $A d S_{5} \times S^{5}$ ", arxiv:1012.3987.

[III.1] M. Staudacher, "Review of AdS/CFT Integrability, Chapter III.1: Bethe Ansätze and the R-Matrix Formalism", arxiv:1012.3990.

[III.3] P. Vieira and D. Volin, "Review of AdS/CFT Integrability, Chapter III.3: The dressing factor", arxiv:1012.3992.

[III.4] L. Freyhult, "Review of AdS/CFT Integrability, Chapter III.4: Twist states and the cusp anomalous dimension", arxiv:1012.3993.

[III.5] R. Janik, "Review of AdS/CFT Integrability, Chapter III.5: Lüscher corrections", arxiv:1012.3994.

[III.6] Z. Bajnok, "Review of AdS/CFT Integrability, Chapter III.6: Thermodynamic Bethe Ansatz", arxiv:1012.3995.

[III.7] V. Kazakov and N. Gromov, "Review of AdS/CFT Integrability, Chapter III.7: Hirota Dynamics for Quantum Integrability", arxiv:1012.3996.

[IV.1] C. Kristjansen, "Review of AdS/CFT Integrability, Chapter IV.1: Aspects of Non-planarity", arxiv:1012.3997.

[IV.2] K. Zoubos, "Review of AdS/CFT Integrability, Chapter IV.2: Deformations, Orbifolds and Open Boundaries", arxiv:1012.3998.

[IV.3] T. Klose, "Review of AdS/CFT Integrability, Chapter IV.3: $\mathcal{N}=6$ Chern-Simons and Strings on $A d S_{4} \times C P^{3} "$, arxiv:1012.3999.

[1] J. M. Maldacena, "The large $N$ limit of superconformal field theories and supergravity", Adv. Theor. Math. Phys. 2, 231 (1998), hep-th/9711200. • S. S. Gubser, I. R. Klebanov and A. M. Polyakov, "Gauge theory correlators from non-critical string theory", Phys. Lett. B428, 105 (1998), hep-th/9802109. • E. Witten, "Anti-de Sitter space and holography", Adv. Theor. Math. Phys. 2, 253 (1998), hep-th/9802150. 
[2] D. Serban and M. Staudacher, "Planar $\mathcal{N}=4$ gauge theory and the Inozemtsev long range spin chain", JHEP 0406, 001 (2004), hep-th/0401057.

[3] N. Beisert, V. Dippel and M. Staudacher, "A novel long range spin chain and planar $\mathcal{N}=4$ super Yang-Mills", JHEP 0407, 075 (2004), hep-th/0405001.

[4] C. Sieg and A. Torrielli, "Wrapping interactions and the genus expansion of the 2point function of composite operators", Nucl. Phys. B723, 3 (2005), hep-th/0505071.

[5] J. Ambjorn, R. A. Janik and C. Kristjansen, "Wrapping interactions and a new source of corrections to the spin-chain / string duality", Nucl. Phys. B736, 288 (2006), hep-th/0510171.

[6] W. Siegel, "Supersymmetric Dimensional Regularization via Dimensional Reduction", Phys. Lett. B84, 193 (1979)

[7] M. T. Grisaru, M. Rocek and W. Siegel, "Superloops 3, beta 0: A Calculation in $\mathcal{N}=4$ Yang-Mills Theory", Nucl. Phys. B183, 141 (1981). • M. T. Grisaru, M. Rocek and W. Siegel, "Zero Three Loop beta Function in $\mathcal{N}=4$ Superyang-Mills Theory", Phys. Rev. Lett. 45, 1063 (1980). • W. E. Caswell and D. Zanon, "Vanishing Three Loop beta Function in $\mathcal{N}=4$ Supersymmetric Yang-Mills Theory", Phys. Lett. B100, 152 (1981). • W. E. Caswell and D. Zanon, "Zero Three Loop beta Function in the $\mathcal{N}=4$ Supersymmetric Yang-Mills Theory", Nucl. Phys. B182, 125 (1981).

[8] S. Mandelstam, "Light Cone Superspace and the Ultraviolet Finiteness of the $\mathcal{N}=4$ Model", Nucl. Phys. B213, 149 (1983) • L. Brink, O. Lindgren and B. E. W. Nilsson, "The Ultraviolet Finiteness of the $\mathcal{N}=4$ Yang-Mills Theory", Phys. Lett. B123, 323 (1983) • P. S. Howe, K. S. Stelle and P. K. Townsend, "The Relaxed Hypermultiplet: An Unconstrained $\mathcal{N}=2$ Superfield Theory", Nucl. Phys. B214, 519 (1983). • P. S. Howe, K. S. Stelle and P. K. Townsend, "Miraculous Ultraviolet Cancellations in Supersymmetry Made Manifest", Nucl. Phys. B236, 125 (1984).

[9] S. Ferrara and B. Zumino, "Supergauge Invariant Yang-Mills Theories", Nucl. Phys. B79, 413 (1974).

[10] C. Sieg, "Superspace calculation of the three-loop dilatation operator of $\mathcal{N}=4 S Y M$ theory", arxiv:1008.3351.

[11] S. J. Gates, M. T. Grisaru, M. Rocek and W. Siegel, "Superspace, or one thousand and one lessons in supersymmetry", Front. Phys. 58, 1 (1983), hep-th/0108200.

[12] J. A. Minahan, "The $S U(2)$ sector in AdS/CFT", Fortsch. Phys. 53, 828 (2005), hep-th/0503143.

[13] N. Beisert, C. Kristjansen and M. Staudacher, "The dilatation operator of $\mathcal{N}=4$ super Yang-Mills theory", Nucl. Phys. B664, 131 (2003), hep-th/0303060.

[14] N. Beisert and T. Klose, "Long-range gl(n) integrable spin chains and plane-wave matrix theory", J. Stat. Mech. 0607, P006 (2006), hep-th/0510124.

[15] F. Fiamberti, A. Santambrogio, C. Sieg and D. Zanon, "Wrapping at four loops in $\mathcal{N}=4$ SYM", Phys. Lett. B666, 100 (2008), arxiv:0712.3522. 
[16] F. Fiamberti, A. Santambrogio, C. Sieg and D. Zanon, "Anomalous dimension with wrapping at four loops in $\mathcal{N}=4 S Y M$ ", Nucl. Phys. B805, 231 (2008), arxiv:0806.2095.

[17] D. E. Berenstein, J. M. Maldacena and H. S. Nastase, "Strings in flat space and pp waves from $\mathcal{N}=4$ super Yang Mills", JHEP 0204, 013 (2002), hep-th/0202021.

[18] J. A. Minahan and K. Zarembo, "The Bethe-ansatz for $\mathcal{N}=4$ super Yang-Mills", JHEP 0303, 013 (2003), hep-th/0212208.

[19] D. J. Gross, A. Mikhailov and R. Roiban, "A calculation of the plane wave string Hamiltonian from $\mathcal{N}=4$ super-Yang-Mills theory", JHEP 0305, 025 (2003), hep-th/0208231.

[20] B. Eden, C. Jarczak and E. Sokatchev, "A three-loop test of the dilatation operator in $\mathcal{N}=4$ SYM", Nucl. Phys. B712, 157 (2005), hep-th/0409009.

[21] D. Anselmi, "The $\mathcal{N}=4$ quantum conformal algebra", Nucl. Phys. B541, 369 (1999), hep-th/9809192.

[22] N. Beisert, "The su(2|2) dynamic S-matrix", Adv. Theor. Math. Phys. 12, 945 (2008), hep-th/0511082.

[23] D. J. Gross, A. Mikhailov and R. Roiban, "Operators with large $R$ charge in $\mathcal{N}=4$ Yang-Mills theory", Annals Phys. 301, 31 (2002), hep-th/0205066.

[24] N. Beisert, B. Eden and M. Staudacher, "Transcendentality and crossing", J. Stat. Mech. 0701, P021 (2007), hep-th/0610251.

[25] G. Arutyunov, S. Frolov and M. Staudacher, "Bethe ansatz for quantum strings", JHEP 0410, 016 (2004), hep-th/0406256. • R. Hernandez and E. Lopez, "Quantum corrections to the string Bethe ansatz", JHEP 0607, 004 (2006), hep-th/0603204.

[26] Z. Bern, M. Czakon, L. J. Dixon, D. A. Kosower and V. A. Smirnov, "The Four-Loop Planar Amplitude and Cusp Anomalous Dimension in Maximally Supersymmetric Yang-Mills Theory", Phys. Rev. D75, 085010 (2007), hep-th/0610248.

[27] A. Santambrogio and D. Zanon, "Exact anomalous dimensions of $\mathcal{N}=4$ Yang-Mills operators with large $R$ charge", Phys. Lett. B545, 425 (2002), hep-th/0206079.

[28] B. Eden, "A two-loop test for the factorised S-matrix of planar $\mathcal{N}=4$ ", Nucl. Phys. B738, 409 (2006), hep-th/0501234.

[29] N. Beisert, T. McLoughlin and R. Roiban, "The Four-Loop Dressing Phase of $\mathcal{N}=4$ SYM", Phys. Rev. D76, 046002 (2007), arxiv:0705.0321.

[30] F. Fiamberti, A. Santambrogio and C. Sieg, "Five-loop anomalous dimension at critical wrapping order in $\mathcal{N}=4 S Y M "$, arxiv:0908.0234.

[31] K. Konishi, "Anomalous Supersymmetry Transformation of Some Composite Operators in SQCD", Phys. Lett. B135, 439 (1984).

[32] D. Anselmi, M. T. Grisaru and A. Johansen, "A Critical Behaviour of Anomalous Currents, Electric- Magnetic Universality and CFT ", Nucl. Phys. B491, 221 (1997), hep-th/9601023. • D. Anselmi, D. Z. Freedman, M. T. Grisaru and A. A. Johansen, "Universality of the operator product expansions of SCFT(4)", Phys. Lett. B394, 329 (1997), hep-th/9608125. 
[33] M. Bianchi, S. Kovacs, G. Rossi and Y. S. Stanev, "On the logarithmic behavior in $\mathcal{N}=4$ SYM theory", JHEP 9908, 020 (1999), hep-th/9906188. M. Bianchi,

S. Kovacs, G. Rossi and Y. S. Stanev, "Anomalous dimensions in $\mathcal{N}=4 S Y M$ theory at order $g^{* *} 4$ ", Nucl. Phys. B584, 216 (2000), hep-th/0003203.

[34] G. Arutyunov, B. Eden, A. C. Petkou and E. Sokatchev, "Exceptional non-renormalization properties and OPE analysis of chiral four-point functions in $\mathcal{N}=4 S Y M(4) "$, Nucl. Phys. B620, 380 (2002), hep-th/0103230.

[35] M. Bianchi, S. Kovacs, G. Rossi and Y. S. Stanev, "Properties of the Konishi multiplet in $\mathcal{N}=4$ SYM theory", JHEP 0105, 042 (2001), hep-th/0104016.

[36] A. V. Kotikov, L. N. Lipatov and V. N. Velizhanin, "Anomalous dimensions of Wilson operators in $\mathcal{N}=4$ SYM theory", Phys. Lett. B557, 114 (2003), hep-ph/0301021.

[37] A. V. Kotikov, L. N. Lipatov, A. I. Onishchenko and V. N. Velizhanin, "Three-loop universal anomalous dimension of the Wilson operators in $\mathcal{N}=4$ SUSY Yang-Mills model", Phys. Lett. B595, 521 (2004), hep-th/0404092.

[38] S. Moch, J. A. M. Vermaseren and A. Vogt, "The three-loop splitting functions in QCD: The non-singlet case", Nucl. Phys. B688, 101 (2004), hep-ph/0403192.

[39] A. V. Kotikov and L. N. Lipatov, "DGLAP and BFKL evolution equations in the $\mathcal{N}=4$ supersymmetric gauge theory", Nucl. Phys. B661, 19 (2003), hep-ph/0208220.

[40] F. Fiamberti, A. Santambrogio and C. Sieg, "Superspace methods for the computation of wrapping effects in the standard and beta-deformed $\mathcal{N}=4 S Y M "$ ", arxiv: 1006.3475 .

[41] T. Fischbacher, T. Klose and J. Plefka, "Planar plane-wave matrix theory at the four loop order: Integrability without BMN scaling", JHEP 0502, 039 (2005), hep-th/0412331.

[42] Z. Bajnok and R. A. Janik, "Four-loop perturbative Konishi from strings and finite size effects for multiparticle states", Nucl. Phys. B807, 625 (2009), arxiv: 0807.0399

[43] V. N. Velizhanin, "The Four-Loop Konishi in $\mathcal{N}=4$ SYM", arxiv:0808.3832.

[44] N. Gromov, V. Kazakov, A. Kozak and P. Vieira, "Integrability for the Full Spectrum of Planar AdS/CFT II", arxiv:0902.4458. • N. Gromov, V. Kazakov and P. Vieira, "Exact Spectrum of Anomalous Dimensions of Planar $\mathcal{N}=4$ Supersymmetric Yang-Mills Theory", Phys. Rev. Lett. 103, 131601 (2009), arxiv:0901.3753.

[45] D. Bombardelli, D. Fioravanti and R. Tateo, "Thermodynamic Bethe Ansatz for planar AdS/CFT: a proposal", J. Phys. A42, 375401 (2009), arxiv:0902.3930 • G. Arutyunov and S. Frolov, "On String S-matrix, Bound States and TBA", JHEP 0712, 024 (2007), arxiv:0710.1568. • G. Arutyunov and S. Frolov, "String hypothesis for the $A d S_{5} \times S^{5}$ mirror", JHEP 0903, 152 (2009), arxiv:0901.1417.

[46] Z. Bajnok, R. A. Janik and T. Lukowski, "Four loop twist two, BFKL, wrapping and strings", Nucl. Phys. B816, 376 (2009), arxiv:0811.4448.

[47] Z. Bajnok, A. Hegedus, R. A. Janik and T. Lukowski, "Five loop Konishi from AdS/CFT", Nucl. Phys. B827, 426 (2010), arxiv:0906.4062.

[48] G. Arutyunov, S. Frolov and R. Suzuki, "Five-loop Konishi from the Mirror TBA", JHEP 1004, 069 (2010), arxiv:1002.1711. • J. Balog and A. Hegedus, "5-loop 
Konishi from linearized TBA and the XXX magnet", JHEP 1006, 080 (2010), arxiv:1002.4142.

[49] T. Lukowski, A. Rej and V. N. Velizhanin, "Five-Loop Anomalous Dimension of Twist-Two Operators", Nucl. Phys. B831, 105 (2010), arxiv:0912.1624.

[50] M. Beccaria, V. Forini, T. Lukowski and S. Zieme, "Twist-three at five loops, Bethe Ansatz and wrapping", JHEP 0903, 129 (2009), arxiv:0901.4864.

[51] V. N. Velizhanin, "Six-Loop Anomalous Dimension of Twist-Three Operators in $\mathcal{N}=4 S Y M "$, arxiv:1003.4717.

[52] V. N. Velizhanin, "Leading transcedentality contributions to the four-loop universal anomalous dimension in $\mathcal{N}=4 S Y M "$, arxiv:0811.0607.

[53] F. Fiamberti, A. Santambrogio, C. Sieg and D. Zanon, "Finite-size effects in the superconformal beta-deformed $\mathcal{N}=4 S Y M "$, JHEP 0808, 057 (2008),

arxiv:0806.2103. • F. Fiamberti, A. Santambrogio, C. Sieg and D. Zanon, "Single impurity operators at critical wrapping order in the beta-deformed $\mathcal{N}=4 S Y M "$ ", JHEP 0908, 034 (2009), arxiv:0811.4594.

[54] M. Beccaria and G. F. De Angelis, "On the wrapping correction to single magnon energy in twisted N=4 SYM", [nt. J. Mod. Phys. A24 5803 (2009) arxiv:0903.0778.

- N. Gromov and F. Levkovich-Maslyuk, "Y-system and beta-deformed $\mathcal{N}=4$ Super-Yang-Mills", arxiv:1006.5438, • G. Arutyunov, M. de Leeuw and S. J. van Tongeren, "Twisting the Mirror TBA", JHEP 1102025 (2011), arxiv:1009.4118.

[55] K. G. Chetyrkin, A. L. Kataev and F. V. Tkachov, "New Approach to Evaluation of Multiloop Feynman Integrals: The Gegenbauer Polynomial x Space Technique", Nucl. Phys. B174, 345 (1980). • A. V. Kotikov, "The Gegenbauer Polynomial Technique: the evaluation of a class of Feynman diagrams", Phys. Lett. B375, 240 (1996), hep-ph/9512270.

[56] D. J. Broadhurst, "Evaluation of a class of Feynman diagrams for all numbers of loops and dimensions", Phys. Lett. B164, 356 (1985). 